\title{
SQUID DKA - baseline audit of diabetic ketoacidosis management at a large NHS foundation trust in the West Midlands
}

\author{
Authors: Punith Kempegowda, Amna Noorin, Ayesha Ishaq, Ateeq Syed and Muhammad Karamat
}

\begin{abstract}
Aims
To study the common precipitating factors and the current practice of diabetic ketoacidosis (DKA) management as part of the service and quality improvement of inpatient diabetes (SQUID) management.

\section{Methods}

The study was conducted at the Heart of England NHS Foundation Trust. All patients discharged with a diagnosis of DKA from April to September 2017 were included in the study. Patients who did not meet the national and/or trust criteria for DKA were excluded from the study. Electronically scanned records from emergency medicine and acute medical units for each episode of DKA were studied to minimise the delay in data aggregation. Socio-demographic data and factors influencing DKA management were collected for all DKA episodes. The data was analysed using IBM's SPSS Statistics statistical analysis software and are expressed in percentage and median with inter-quartile range (IQR) as appropriate.
\end{abstract}

\section{Results}

A total of 146 DKA episodes were studied. Four episodes were excluded from final analysis. 64.8\% (92/142) episodes were correctly diagnosed with DKA (male:female 1:1). The median and IQR age was 33.3 (23.0-52.1) years. 19.8\% (18/92) had type 2 diabetes. Suboptimal compliance with diabetes therapy (58.7\%) followed by inter-current illness (30.4\%) were the most common precipitating factors for DKA. The average delay for treatment initiation following diagnosis of DKA was 0.8 hours. The median DKA duration, length of stay, hourly glucose and hourly ketone monitoring were 18.0 (12.0-28.0) hours, 45.0 (23.0-116.8) hours, $61.9 \%(45.5-107.1)$ and $58.3 \%$ (39.1-107.1), respectively. The time to background insulin administration following the diagnosis of DKA was 16.0 (6.5-23.5) hours. The median time to referral to specialist review was 13.0 (4.5-20.8) hours. Following referral, patients were reviewed in $2.0(1.0-19.3)$ hours.

Authors: Department of Diabetes and Endocrinology, Heart of England NHS Foundation Trust, Birmingham, UK

\section{Conclusion}

We note room for improvement of DKA management at our trust. A significant variation was also observed in the management of DKA episodes. These results are discussed with all stakeholders across the trust and various interventions are initiated to improve the DKA management. Further mediations will be based on the results from ongoing regular audits to provide the best and uniform care for DKA across the trust.

\section{Conflict of interest statement}

None. 